\title{
Energy Efficiency Behavior in Heterogeneous Networks under Various Operating Situations of Cognitive Small Cells
}

\author{
Amr A. Fahmy1, Asmaa M. Saafan², Hesham M. El-Badawy², Salwa El-Ramly ${ }^{1}$ \\ ${ }^{1}$ Faculty of Engineering, Ain-Shams University, Cairo, Egypt \\ ${ }^{2}$ National Telecommunication Institute (NTI), Cairo, Egypt \\ Email: amrofahmy@gmail.com, asmaa.nti@gmail.com, heshamelbadawy@ieee.org, \\ salwa elramly@eng.asu.edu.eg
}

Received 30 December 2014; accepted 16 January 2015; published 19 January 2015

Copyright @ 2015 by authors and Scientific Research Publishing Inc.

This work is licensed under the Creative Commons Attribution International License (CC BY). http://creativecommons.org/licenses/by/4.0/

(c) $\underset{\mathrm{EY}}{\mathrm{B}}$ Open Access

\section{Abstract}

Recently, several approaches were followed for the enhancement and better resource utilization in mobile networks; this is to achieve energy efficient consumption for production and delivery of an information bit. Using Cognitive Femto cells (as a member of the small base stations' family) proves that, it is an efficient solution for achieving this goal [1]. The use of Energy Efficiency term $(\eta)$ has become one of the major indices for measuring the performance of these systems. $\eta$ is the measure of the overall system Capacity $(C)$ in bps/Hz versus the Consumed Energy $(E)$ in Joules [2]. In consistence with many researches, analytic models and empirical measurements, $\eta$ will be investigated throughout the course of this work. Cognitive Base Stations (CBS) (as an element of the system model) which performs the traffic offloading operations is proved to enhance $\eta$ performance. In this work, a combination of both analytic and simulation models are used to construct a practical system model. The obtained model is then used to illustrate the effect of different operational parameters that are involved in the $\eta$ problem. On the other hand, the current paper tries to focus on the selection criteria that may be used to design the cooperative cognitive networks in order to achieve the best $\eta$ indices. Both of CBSs radii as well as the inter-separation distances (between CBSs and MBS location) are examined to obtain best $\eta$ index for different operation scenarios; in addition, both of capacity and energy consumption are taken into consideration based on practical operating measures. This work proposed several nonlinear equations with fixed parameters to be used by field engineers to achieve the results with minimum reduced computation complexity. So, the current work may be of importance for the regulator bodies as well as the cognitive mobile operators. 


\section{Keywords}

\section{Energy Efficiency, Capacity, Energy, Cognitive Radio, Wireless Communications, Green Radio}

\section{Introduction}

Recently, huge interest has evolved for finding means to conserve the natural resources especially in communications. Two facts are realized by communication systems' engineers and researchers: the rapid consumption of natural resources and the increase in global warming with the dependency growth of civilizations on energy for using wireless communication systems, together, the scarcity of spectrum resources of these wireless communication systems. "Green Communication" is currently one of the hottest topics in the field of wireless communication researches due to its contributions in saving the environment [3] [4]. "Energy Efficiency $(\eta)$ " is a major index suggested by EARTH's Energy Efficiency Evaluation Framework $\left(\mathrm{E}^{3} \mathrm{~F}\right)[5]-[8]$; it is pursuing methods for saving energy consumed in accomplishing communications, which reduces pollution and preserves natural resources. Forthcoming mobile Generations will face many tradeoffs between the $\eta$ considerations versus system performance parameters especially throughput. Cognitive Radio (CR) is one of the most elected techniques to be recognized as a candidate technology to implement Heterogeneous Networks (HetNet) Communications with better $\eta$. This paper illustrates different aspects that affect the $\eta$ behavior. These parameters include Cognitive Base Stations (CBS) radii (which will directly affect the CBS coverage area) and the distance between these CBSs from Macro Base Stations (MBS). A combination of analytical and simulation methods has been adopted to accomplish the aimed target of the current work. Simulation scenarios are performed to illustrate the contribution of these parameters in the whole system Energy Efficiency.

The paper is organized as follows: Section 2 will introduce the CR based HetNet and its relation to the $\eta$ index for CR networks. Section 3 proposes a system model and assumptions that will be adopted to evaluate the $\eta$ index analytically. Whereas, Section 4 presents the performance evaluation and model validation, in which, main design milestones for the different simulation scenarios are illustrated. Section 5 previews the obtained results and give detailed analysis about these results. Finally, Section 6 concludes the paper and gives the future directions for such area of interest.

\section{Literature Review}

In CR based HetNets, Green communications has become a hot topic in the wireless telecommunication era. EARTH is one of the major European research projects that is concerned about finding energy efficient solutions by the reduction of the overall energy consumption [9]. EARTH proposes that mobile communications can achieve a reduction of about 50\% of its concurrent energy consumption [1]. In 2012, Energy Efficiency Evaluation Framework $\left(\mathrm{E}^{3} \mathrm{~F}\right)$ has been established to find out means to increase the radio network's energy efficiency. In $1^{\text {st }}$ ETSI TC EE workshop [10], EARTH $E^{3} F$ builds on the 3GPP evaluation framework for LTE [11]. In frame of these resources, many researches have evolved, but this work focuses on a few needed here. In [12], necessary enhancements over existing performance evaluation frameworks were discussed, such that the energy efficiency of the entire network comprising component, node and network level contributions were quantified. Importantly, a power model for various BS types were suggested, in which it correlates between the desired radiated power and its requirements from the power feed/supply point of view. The mentioned proposed evaluation framework is applied to quantify the energy efficiency of the downlink of a 3GPP LTE radio access network. In [13], a proposal is offered of an analytical model for Cognitive Femto Network (CFN) deployed in HetNet, and, an evaluation is done for the impact of CFN usage on energy consumption discussing the relevant tradeoffs and practical issues. This work led to finding that CFN is beneficial for improving the Energy Efficiency $(\eta)$. Also, that the resultant $\eta$ gains are dependent on factors entailing CBS deployment density and usage rate, sensing period selection and interference management. Reference [14] discussed scheduling in CR networks (CRNs), in which, cognitive base station (CBS) makes frequency allocations to the CRs at the beginning of each frame. It also stated that a cognitive scheduler must consider the diversity among CRs' queues and channel capacities in terms of number of bits as well as the channel switching cost from one frequency to another. 


\section{System Model and Assumptions}

The system model elements are shown in Figure 1, a Macro-Base Station (MBS) is situated at the origin of the system, this MBS is assumed to cover a circular area, MBS operation parameters are shown later in Section 4. The downlink channel is assumed to follow the space loss model such that [15]:

$$
P_{r}=P_{\text {out }} d^{-\alpha}
$$

where, $P_{r}$, is the received power at the receiver side, $P_{\text {out }}$, is the antenna output radiated power at the transmitter side, $\alpha$, is the free space path loss exponent, $d$ is the distance between the transmitter and receiver antennas.

User Equipments (UE) joining this system model are either Macro Users (MU), where they are served by the MBS, or, Cognitive Users (CU) where they are served by the nearest CBS. They are all deployed uniformly in the MBS coverage area (locations are shown as “+” sign). $N_{\mathrm{UE}}$ is the total number of UEs, and is comprised of $N_{\text {MU }}$ and $N_{\text {CU }}$ users and are shared between MBS and CBSs respectively. Those UEs are assumed to be stationary (no mobility models used). Operational parameters of those UEs (MU or CU) are shown later in Section 4. CBSs are deployed uniformly at equal distances $d$ from MBS situated at the origin of the system, this equal distance is meant to simulate the average (mean) distance for those CBSs uniformly distributed over MBS coverage area, in addition, their coverage areas are not supposed to overlap. The number of CBSs $\left(n_{C}\right)$ depends on how far from MBS they are situated, and, their cell coverage radii $R_{c}$. The larger $R_{c}$ and the shorter $d$, the smaller number of CBS deployed and vice-versa. The non-overlapped CBS will give the nearest resemblance to those CBSs deployed over a Voroni-tessellation scheme [16] [17]. A single Primary User (PU) at the MBS cell edge is considered, this PU will perform the interference as an adjacent cell interferer to MBSs' frequency. This PU no traffic activity probability $P_{\text {idle }}$ equals 0.4. Interference among system elements is assumed too; there are several interference types that are illustrated and considered in the next section. Moreover, $R_{c}$, $d, n_{C}$, will be varied through the simulation processes to find those major effects over the system $\eta$ components. This assumed model was previously-more or less-introduced and used by multiple works [12] [18]. For model realization (without loss of generality) no resource management system will be applied. In addition, no legacy femto cells exist within the same MBS coverage area. There are $F_{M}$ frequencies provided by MBS for

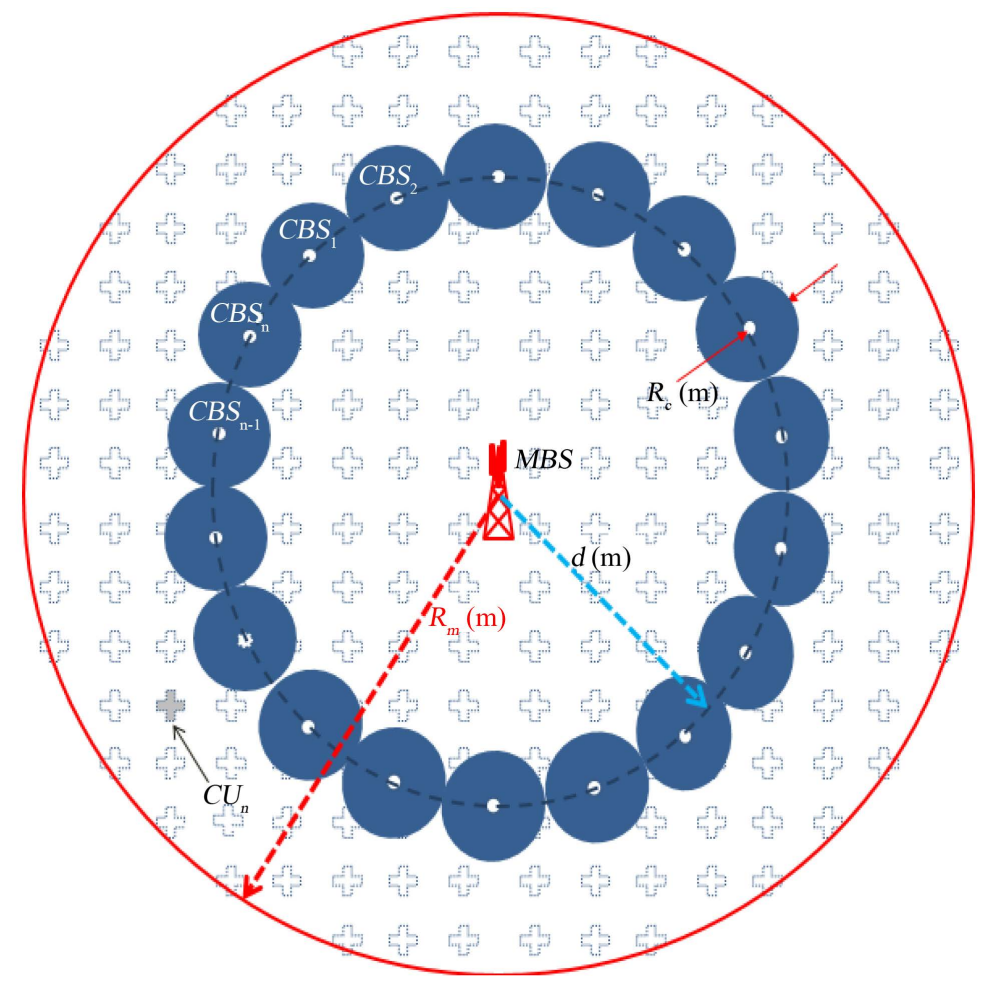

Figure 1. System model. 
usage by UEs, those UEs go through two states of operation, active and idle with active probability $\lambda$. For MBS, and in order to apply $\eta$ concepts, we need to reduce its radiated power to the needed level, i.e., the output power depends on the traffic load (active macro-users $n_{m}^{\text {active }}$ ), such that:

$$
P_{M}^{\text {tx }}=P_{M}^{\text {out }}\left(n_{m}^{\text {active }} / n_{m}^{\max }\right)
$$

where, $P_{M}^{t x}$ is the radiated power depending on the linked active UEs, $P_{M}^{\text {out }}$ is the maximum radiated power, $n_{m}^{\max }$ is the maximum allowable served users by MBS. In this work $n_{m}^{\max }$ is assumed to be 300 users. For CBSs, the operational parameters used are given in Section 4. In [14], Channel switching cost is a linear function of the difference between the current and the "be switched to" frequencies. The average number of channel switching $\delta_{F}$ is involved in calculating the cost of RF antenna tuning (channel switching cost). Here in this work (Section 3.3) it is assumed to be equal to 5. All the parameters used in this work are considered based on the available knowledge of the practical deployment in the urban areas. Also, these parameters are in consistence with [13]. For channel modeling parameters, the space path loss exponent $\alpha$ for communication channels between macro-affiliated system components and cognitive-affiliated system components (i.e. cross layer communications and interference) are assumed to be equal to 2.8, while in mutual layer (co-layer) communication and interference among cognitive-affiliated system components $\alpha$ is assumed to be equal to 2 . This path-loss exponent difference is due to the different nature of working environment of both cases (MBS-UE, or, CBS-UE).

\subsection{Capacity}

Theoretically, capacity $C=R / W$, where $R$ is the bit rate and $W$ is the used bandwidth. Basically, $C$ is calculated using Shannon's theorem which states that [19] [20]:

$$
\frac{R}{W}=\log _{2}(1+\mathrm{SNR})
$$

In this work, theoretical capacity is used to obtain the maximum capacity available by the system, further modifications may be applied to enforce conditions of the actual bit rate. $R$, is influenced by the received signal to noise ratio, the noise is also influenced by the received interference from other system components operating within the same frequency band.

\subsection{Noise and Interference}

In order to calculate the system capacity $C$, the different types of interference should be considered. Assuming that the system only consists of MBSs, CBSs, and a single PU located at the MBS cell-coverage edge, interference is limited to three types of interference, co-layer, cross-layer, and cognitive-layer [13]. The co-Layer Interference occurs when a CBS transmitted signal extends to an adjacent CBS cell causing interference to the neighbor CU operating at the same frequency. The cross-Layer Interference happens in the downlink between macro layer and cognitive layer, that is, when a MBS generates interference to the user receiving at the same frequency in the other layer (i.e. MBS to the CUs and CBS to the MUs). Finally, cognitive Layer Interference where CBS may receive severe interference from the external primary networks at small cell CR frequencies. This interference effect is significantly high in case of misdetection compared to the opportunistic use of the spectrum after successful discovery of the idle bands. In this work, interference is calculated as received signals' strength from source BS to other BSs' UEs. Signals received from other BS are subjected to the space loss model mentioned before , moreover noise spectral density is calculated in its primitive form as:

$$
N_{0}=k \cdot T, \quad \mathrm{~J} / \mathrm{K}
$$

where, $k$ is Boltzmann constant, $T$ is the absolute temperature in $K$, and assuming the involved band width to be $10 \mathrm{MHz}$ (in conformance with LTE standards [15] [21]). Signal to interference ratio is calculated by dividing the received signals' strength $P_{R x}^{\text {dis }}$ at BS/user dis operating at the same frequency by the interference plus noise, such that [15]:

$$
P_{R x}^{\mathrm{dis}}=P_{T x}^{\mathrm{src}} \cdot G^{\mathrm{sc}, \mathrm{dis}}
$$

where, src stands for "source" and dis stands for "destination", and $P_{T x}^{\mathrm{src}}$ is the transmitted signal power from 
transmitter src, $G^{\text {src,dis }}$ is the channel fading gain between a source transmitter src and distant receiver dis due to a space loss model mentioned in (1). Following the analytical model in [13], the interference from multiple sources to a certain destination $I_{\text {dis }}$ is the summation of all the received signals at the distant receiver (and within the same operational bandwidth) multiplied by the activity probability such that:

$$
I_{\text {dis }}=\sum_{\left(n_{\text {src,dis }}=1\right)}^{N_{\text {src, is }}} \lambda_{\text {src }} P_{T x}^{n_{\text {src, dis }}} G^{n_{\text {srr, dis }}}
$$

where, $N_{\text {src,dis }}$ is the number of all active interference sources src causing interference to a single destination dis. Let $F_{\text {src }}$ bethe total number of frequencies provided by src type BS, and $\lambda_{\text {src }}$ be the probability of CU being in active state, thus, the number of interferers as $\lambda_{\text {src }}\left(N_{\text {src }} / F_{\text {src }}\right)$ where $N_{\text {src }}$ is the number of similar type nodes excluding the node itself. CBSs conduct a sensing procedure to allocate usable holes in the spectrum, the sensing period is performed once in each $T_{s}$ time slots. The a detection probability $p_{d}$ during this period is $p_{d}\left(T_{s}\right)$. So, by suggesting that capital letters $C, M$ denote CBSs and MBS, and small letters $c, m, p$ denote CU, MU and PU respectively, and in consistence with [13]:

$$
\begin{gathered}
I_{m}=n_{C, m} I_{C, m}+N_{0}, \\
I_{c}=n_{C, c} I_{C, c}+n_{M, c} I_{M, c}+n_{p, c}\left(1-p_{d}\left(T_{s}\right)\right) I_{p, c}+N_{0},
\end{gathered}
$$

where, $I_{m}, I_{c}$ are the total interference at all users of macro and Cognitive stations respectively (either MU or $\mathrm{CU})$. Assuming a probability of detection and false alarm for MBS frequencies within time slots $T_{s}$ are [13]:

$$
\begin{aligned}
& p_{d}\left(T_{s}\right)=0.9 /\left(T_{s}-1\right) \\
& p_{f a}\left(T_{s}\right)=0.1\left(T_{s}-1\right)
\end{aligned}
$$

Which are in consistence with [13]. Let $F_{M}, F_{C}$ be the number of frequencies provided by MBS, CBS respectively, and $n_{m}, n_{c}$ as the number of MUs, CUs respectively, it is now possible to get the obtained capacity of the various system components $\mathrm{MU}, \mathrm{CU}$, where:

$$
\begin{gathered}
C_{m}=\frac{F_{M}}{n_{m}} \log _{2}\left(1+\frac{P_{m}^{r x}}{I_{m}}\right) \\
C_{c}=\frac{T_{s}-1}{T_{s}} \cdot \frac{F_{C}}{n_{c}} \log _{2}\left(1+\frac{P_{c}^{r x}}{I_{c}}\right) .
\end{gathered}
$$

where, $P_{m}^{r x}, P_{c}^{r x}$ are the received power at MU, CU from their affiliate MBS, CBS (respectively). Thus, the overall capacity of the system model components is:

$$
C=C_{m}+C_{c}
$$

\subsection{Energy Consumption Estimation}

To evaluate the overall consumed energy in the proposed model, all the major system components are considered. In [12], those components are put in a certain framework to simulate the relevant aspects of the Radio Access Network (RAN) at system level. The candidate framework is based on an outcome of thorough work from standardization bodies such as 3GPP [11], international research projects such as the EU project Wireless World Initiative New Radio (WINNER) [22], partners from academia and industry, the global effort in ITU to evaluate system proposals for compliance with IMT-Advanced requirements [23]. EARTH- $\mathrm{E}^{3} \mathrm{~F}$ builds on the 3GPP evaluation framework for LTE based on a sophisticated power model. This framework considers two main measures, the base station (MBS, CBS) power consumption and user equipment (MU, CU) power consumption. Figure 2, demonstrates the input power consumed by MBS system components versus output RF power percentage. The main governing parameters in this framework are: mains power supply (MS), cooling system power consumption (CO), DC to DC converters (DC), baseband processor (BB), RF transceiver (RF), power amplifier (PA). BSs transceivers' components consist of an Antenna Interface (AI), a Power Amplifier (PA), a Radio Frequency (RF) small-signal transceiver section, a baseband (BB) interface including a receiver (uplink), transmitter section (downlink), a DC-DC power supply, an active cooling system, and an AC-DC unit 


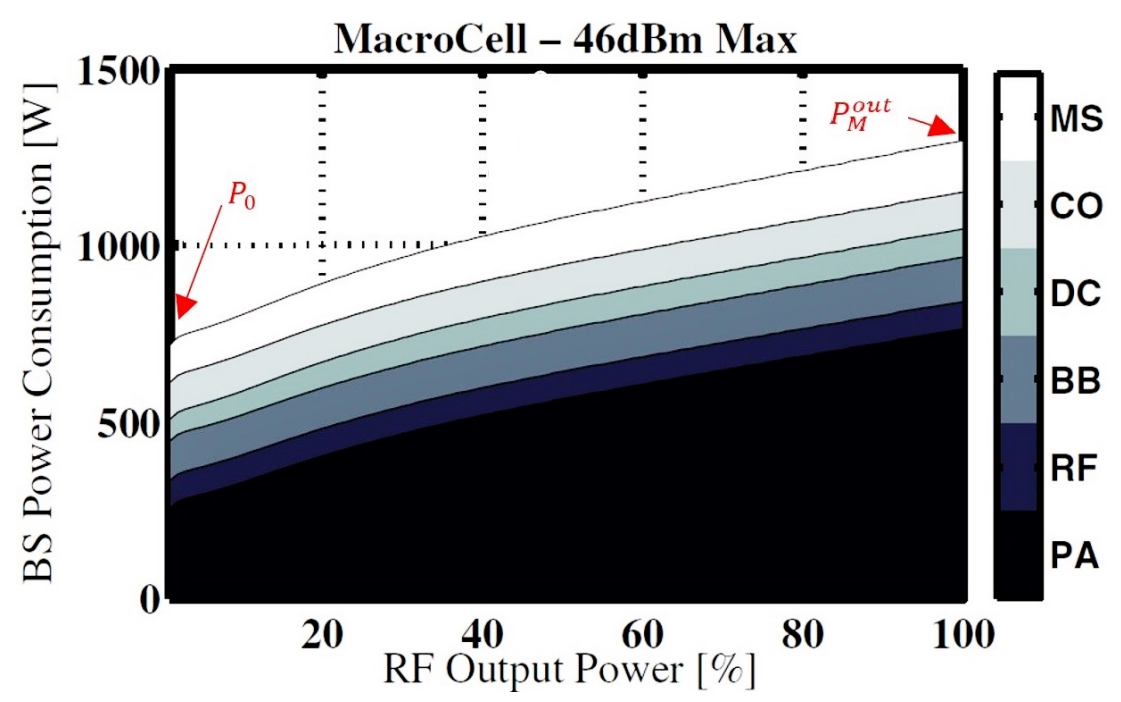

Figure 2. Input power consumed by MBS system components Vs output RF power percentage, considering: mains power supply (MS), cooling system (CO), DC-to-DC converters (DC), baseband processor (BB), RF transceiver (RF), power amplifier (PA) [12].

(mains supply) for connection to the electrical power grid.

For BS Variable Load, (i.e. the power consumption of PA depends on the traffic load), it was found that BS power consumption model is shown in [12], or, an acceptable approximation for the power consumed by MBS is mathematically taken as a straight-line equation by:

$$
P_{M}^{\text {in }}=N_{\text {TRX }} \cdot\left(P_{M}^{0}+\Delta_{p} P_{M}^{\text {out }}\right), \quad P_{M}^{0} \leq P_{M}^{\text {out }} \leq P_{M}^{\max }
$$

where, $P_{M}^{\text {in }}$ denotes the overall power consumed by MBS, $P_{M}^{\max }$ is the maximum RF output power at maximum load, $P_{M}^{0}$ is the power consumption calculated at the minimum possible output power (assumed to be $1 \%$ of $P_{M}^{\max }$ ), and $\Delta_{p}$ is the slope of the load dependent power consumption, $N_{\mathrm{TRX}}$ is the number of TRX chains. For MUs, power consumption depends on the traffic activity condition.

Assuming $P_{m}^{i}$ is the power consumption during idle state, $\lambda_{m}$ is the active downlink probability, and $p$ is the probability success to have an available (operative) frequency from MBS where $p=F_{M} / n_{m}$ (for, $\left.F_{M} \leq n_{m}\right)$. So, CU equipment energy consumption $E_{m}$ is:

$$
E_{m}=\lambda_{m} p P_{m}^{r x}+\left(1-\lambda_{m} p\right) P_{m}^{i}
$$

\subsubsection{Energy Consumption of CBSs}

Figure 3 shows the different states of power consumption by a CBS. In an observation of a number of multiple normalized time slots $T_{s}$. A CBS functions in one of three modes, in the first mode, which is performed in a number of time slots $\tau$, a CBS consumes power $P_{C}^{\text {st }}$ to perform sensing peration for frequency holes with the maximum detection probability. In the second mode, which is performed during the remaining part of the available time slots (i.e. $T_{s}-\tau$ ), a CBS transmits power $P_{C}^{t \mathrm{tt}}=P_{C}^{t x}+P_{C}^{b h}$ to a CU, where $P_{C}^{t x}$ is the consumed power for performing transmission to CUs, and $P_{C}^{b h}$ is the consumed power to handle the backhaul requirements. In the third mode, which is alternatively performed with the second mode and during the other part of $T_{s}-\tau$ slots, a CBS consumes power $P_{C}^{i}$ during a state of idle. Let $\lambda_{c}$ be the probability of a CBS being in the transmit state, so, the average energy consumed during $T_{s}$ is:

$$
E_{C}=\frac{P_{C}^{s t} \tau+\left(\lambda_{c} P_{C}^{\mathrm{txt}}+\left(1-\lambda_{c}\right) P_{C}^{i}\right)\left(T_{s}-\tau\right)}{T_{s}} .
$$

\subsubsection{Energy Consumption of CUs}

Figure 4 shows the different states of power consumption by a CBS. In the same observation of the number of 


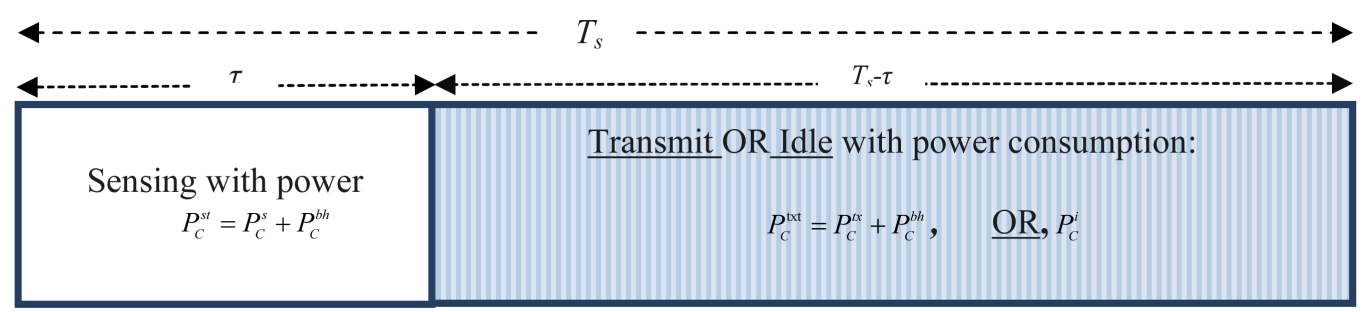

Figure 3. States of energy consumption for CBSs.

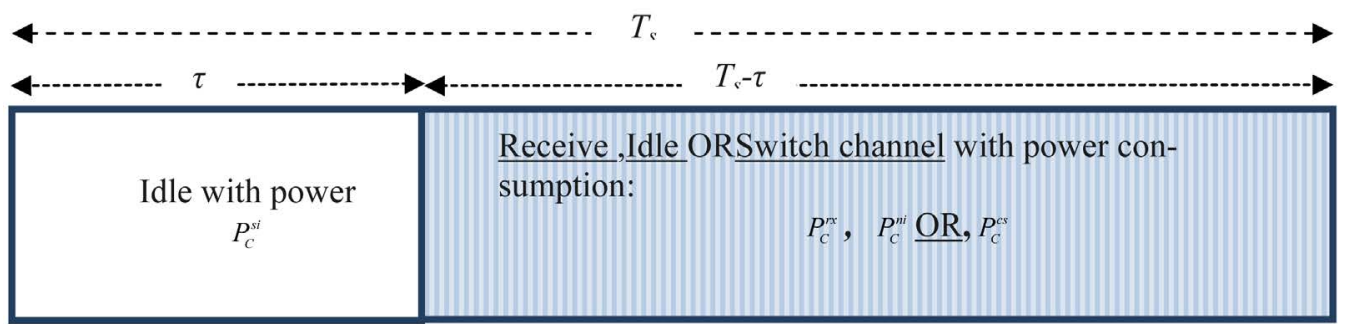

Figure 4. States of energy consumption for CUs.

multiple normalized time slots $T_{s}$, a CU functions in one of three modes. The first mode occurs in a number of time slots $\tau$, in which CBS performs sensing. CU consumes power to perform idle state $P_{c}^{s i}$ during a "CBS sensing period". In the rest of the time slots $T_{s}$ (i.e. $T_{s}-\tau$ ), CU performs reception operation (traffic state) by consuming power $P_{c}^{r x}$. Allocated frequencies by CBS change with an average number of channel switching $\delta_{F}$, it takes $P_{c}^{c s}$ power to perform this channel switching. In the third mode which occurs during the same interval of time $\left(T_{s}-\tau\right)$, CU consumes power $P_{c}^{n i}$ during other "normal" states of idle when not in reception mode.

Let $\lambda_{c}$ be the probability of a CU being in the traffic state, so, the average energy consumed during $T_{s}$ is:

$$
E_{c}=\frac{P_{c}^{s i} \tau+\left(\lambda_{c}\left(P_{c}^{r x}+\delta_{F} P_{c}^{c s}\right)+\left(1-\lambda_{c}\right) P_{c}^{n i}\right)\left(T_{s}-\tau\right)}{T_{s}}
$$

The total energy consumption by the system model is:

$$
E=E_{M}+n_{m} E_{m}+n_{C} E_{C}+n_{c} E_{c},
$$

where, $E_{M}=P_{M}^{\text {in }} T_{s}, n_{m}$ is the number of MUs, $n_{C}$ is the number of CBSs, $n_{c}$ is the number of CUs.

\subsection{Energy Efficiency Estimation}

Using Equations (13) and (18), energy efficiency $\eta$ is calculated as:

$$
\eta=C / E
$$

And since $C$ is in bits/second/Hz, and $E$ is in Joules, so, $\eta$ is in bps/Hz/J.

\section{Performance Evaluation and Model Validation}

\subsection{Proposed Algorithm and Parameters}

Numerical results are obtained using an algorithm which contains the previously mentioned analytical-based functions. Those functions calculate the overall system $\eta$ under several operational conditions. The main features of the simulator design are presented in the flow chart and pseudo code shown in Figure 5. These functions provide the ability to change the main parameters such as the CBS cell radius (coverage distance) $R_{c}(\mathrm{~m})$, the average distance $d(\mathrm{~m})$ between the origin of the model where MBS is located and all MBSs' locations, and the total number of users $N_{\mathrm{UE}}$ served either by MBS or CBSs. All $N_{\mathrm{UE}}$ are located within MBS coverage area, and they are uniformly deployed in this area. Also, CBSs are located at equal distances and uniformly deployed over a circle with radius $d$. All CBSs cells' are deployed so their coverage areas will not intercept. 


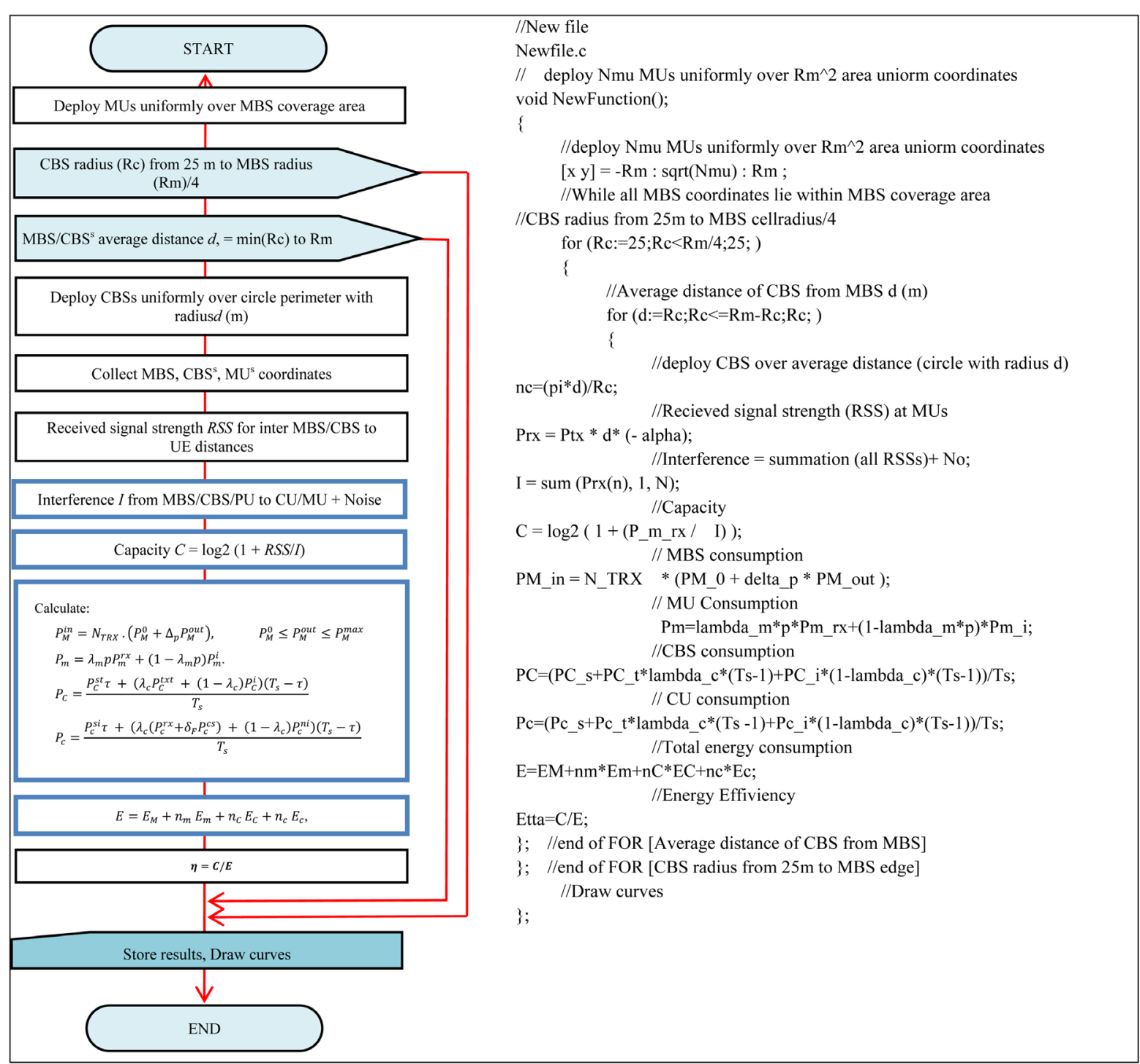

Figure 5. A flow chart and pseudo code that illustrate the main features of the simulation algorithm.

\subsection{Procedures}

The assumed parameters are initialized with values shown in Table 1 and Table 2. Table 1 shows the MBS, UE, CBS assumed values of operating parameters. The average distance between CBSs and MBS $d$ is varied at equal steps between $25 \mathrm{~m}$ and $R_{m}$ (MBS cell radius). The smaller the average distance $d$ the smaller the number of deployable CBSs because their coverage areas should not intercept. In addition, increasing $R_{c}$ will lead to decreasing the number of CBSs deployed over the same $d$. To calculate the power consumed by MBS at variable load $P_{M}^{\text {in }}$, Equation (14) is used by substituting values from Table 2 which shows the assumed parameters to approximate the power consumption behavior of MBS. In this work it is assumed that CBS will operate at a fixed value of $P_{C}^{\text {out }}=0.05 \mathrm{~W}$.

\section{Results}

Figure 6 shows that the number of CBS increases w.r.t. the average distance $d$. It also shows that increasing the CBS cell coverage area (radius) decreases number of CBSs for a given average distance $d$. Geometrically, it is not possible to locate more than two non-overlapped CBSs for the minimum $d \leq R_{c}$. Reasonable coverage radii is considered for CBSs, it is suggested that the minimum cognitive cell coverage radius to be $R_{c} \geq 25 \mathrm{~m}$. This 
Table 1. Assumed operating parameters [13].

\begin{tabular}{cccc}
\hline Model element & Parameter & Explanation & Value \\
\hline \multirow{2}{*}{ MBS } & $\mathrm{R}$ & Radius of cell & $500 \mathrm{~m}$ \\
& $P_{M}^{\text {out }}$ & Max transmission power & $46 \mathrm{dBm}$ \\
& $\mathrm{FM}$ & Number of frequencies & $200 \mathrm{~mW}$ \\
\hline $\mathrm{PE}$ & $P_{m}^{i}$ & Idle state consumption power & $600 \mathrm{~mW}$ \\
& $P_{m}^{r x}$ & Receiving state consumption power & 0.6 \\
& $\lambda_{m}$ & Probability of active traffic state for MU & 0.6 \\
\hline$\lambda_{c}$ & Probability of active traffic state for CU & $30 \mathrm{dBm}$ \\
& $P_{C}^{\text {out }}$ & Max transmission power & $500 \mathrm{~mW}$ \\
& $P_{C}^{i}$ & Consumed power in idle state & $100 \mathrm{~mW}$ \\
& $P_{C}^{b h}$ & Consumed power for backhauling & $600 \mathrm{~mW}$ \\
& $P_{C}^{s}$ & Consumed power in sensing state & 5 \\
& $\delta_{F}$ & Average number of channel switching & 5 \\
\hline
\end{tabular}

Table 2. Power model parameters for MBSs.

\begin{tabular}{ccccc}
\hline$N_{\mathrm{TRX}}$ & $P_{M}^{\text {out }}[\mathrm{W}]$ & $P_{M}^{0}[\mathrm{~W}]$ & $\Delta_{p}$ & 2.66 \\
\hline 6 & 40.0 & 118.7 & 2.7 \\
\hline
\end{tabular}

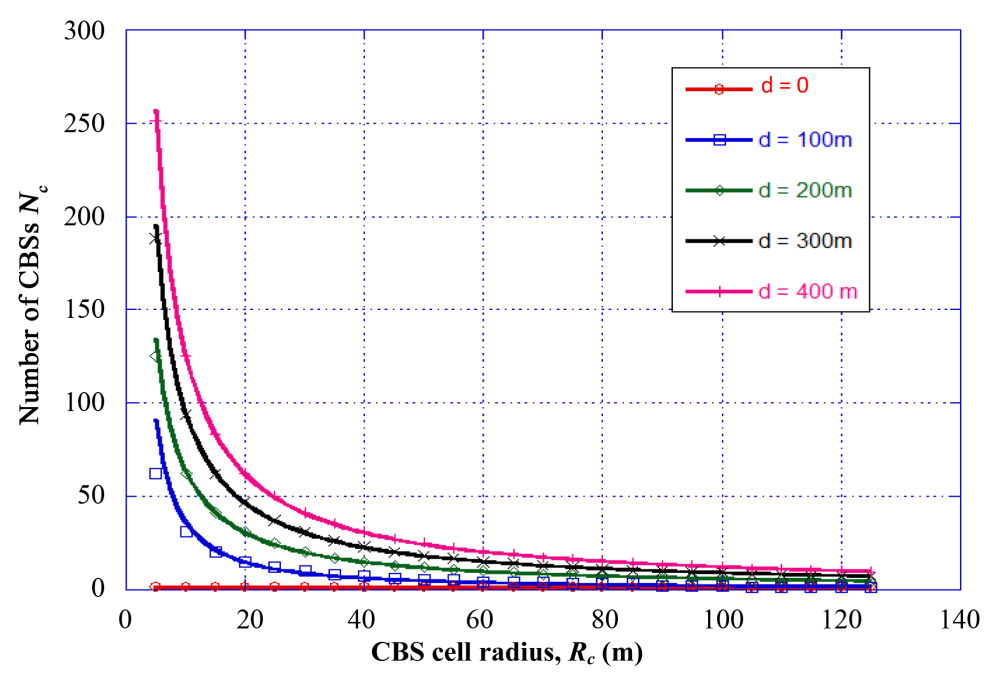

Figure 6. Relation between the number of non-overlapped CBSs $\left(N_{c}\right)$ against cell radius $\left(R_{c}\right)$, for different values of the average distance between CBSs and MBS $(d)$.

figure is useful for converting CBS number $N_{c}$ to average distance $d$ and vice versa, because, in the following figures only $d$ is used, and there will be no need to calculate the number of $N_{c}$. The minimum distance accepted is when CBS cell edge touches the origin, i.e. $\left(d_{\min }=R_{c}\right)$

\subsection{Effect of Inter-Cell Separation Distance}

Figure 7(a) shows the behavior of the proposed model capacity $C$ against $d$ for different values of $R_{c}$. The figure also shows that $C$ increases almost exponentially until it reaches an inflection where it turns to a decrease; this turning point is near the cell edge. The inflection point happens when the number of CBS increases 


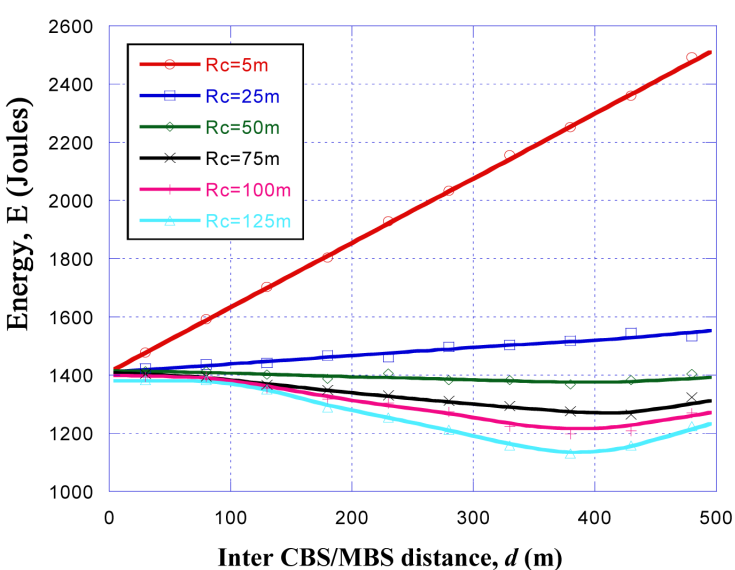

(a)

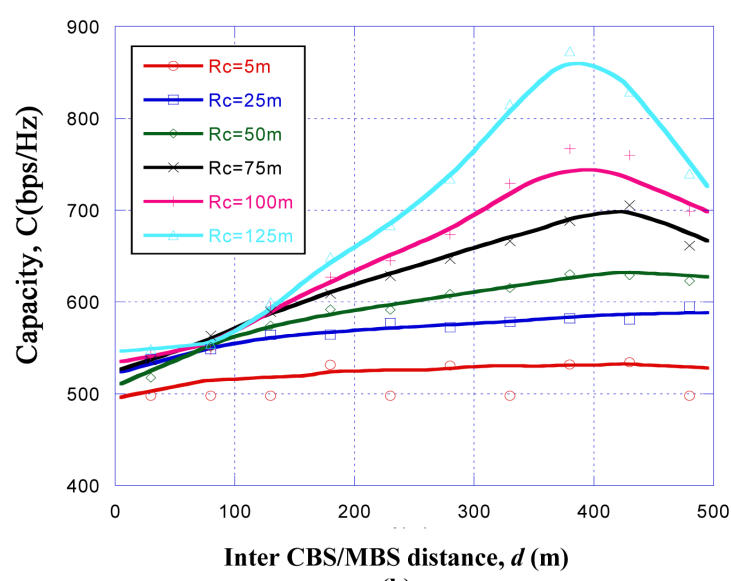

(b)

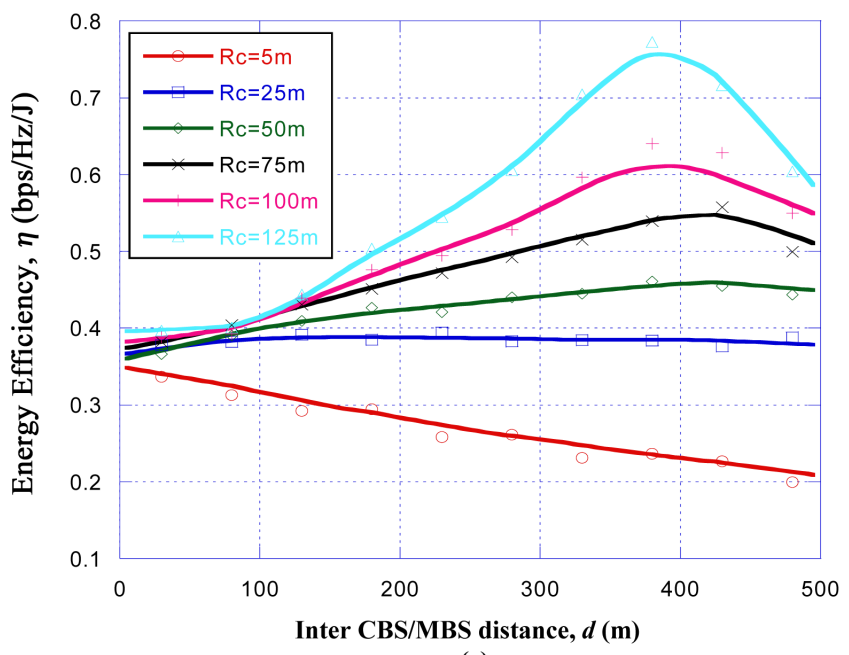

(c)

Figure 7. Behavior of: (a) capacity; (b) energy; (c) energy efficiency, energy efficiency $\eta$ vs. distance $d$ for different values of CBS radii $R_{c}$.

due to increasing the available area for deploying this large number of CBSs (due to reduction of each cell's coverage area). This will lead to more interferers to cause this capacity decay. The "turning point" that happens for different values of $R_{c}$ and at different values of $d$, is found to cause $C$ to act in a more sensitive way to varying $R_{c}$. Approaching the cell edge using CBS with larger coverage areas makes it more likely to find this turning point at larger $d$ and vice versa. "The turning point" exists when $R_{c}=25 \mathrm{~m}$ at $d \cong 380 \mathrm{~m}$, meanwhile, it exists when $R_{c}=100 \mathrm{~m}$, at $d \cong 440 \mathrm{~m}$. Figure 7(b) shows the behavior of the model energy consumption $E$ when varying $d$ for different values of $R_{c}$. It shows that for smaller values of $R_{c}$, the larger the $d$, the larger the $E$ consumption (i.e. no energy is preserved).Energy consumption responds in a less sensitive way to $d$ when $R_{c}$ is increased to values of $R_{c}=40$ meters. Increasing $R_{c}$ over 40 meters then will cause $E$ to be more sensitive to increasing $d$ and will cause energy consumption gain. A turning point from energy gain increase to decay of $E$ is found to happen near the cell edges. Its location $d$ depends on the value of $R_{c}$. It is found that the best energy consumption (gain/preservation) occurs for $R_{c}=125 \mathrm{~m}, d \cong 390 \mathrm{~m}$. Figure 7(c), shows the behavior of Energy Efficiency $\eta$ (bps/Hz/J) for different values of $d(\mathrm{~m})$ at several values of $R_{c}(\mathrm{~m})$. It is found that $\eta$ increases with the increase of $d$ till it inflects and starts decreasing due to the increase of deployable number of CBSs at a specific inter-MBS/CBS cell distance $d$ (where $N_{c} \cong\left\lfloor\pi d / R_{c}\right\rfloor$ ). Energy efficiency $\eta$ is more sensitive to the larger values of $d$, and larger values of $R_{c}$. Those peaks (maxima) found in the previously mentioned results happen at different situations depending on the values of $d$ and $R_{c}$. This is tempting to investigate the behavior of those peaks (maxima). In another numerical approach, the maxima of the obtained results are shown in Figure 8, the maximum values of $C$, $E$, and 
$\eta$ were investigated by filtering maximum values of $C$, and plotting those maxima against the whole range of $d$ and at different values of $R_{c}$ in Figure 8(a). The results show that increasing $R_{c}$ yields to an increase in maximum values of $C$ (i.e. more radio resources per unit area). This is due to the increase in offloaded traffic from MBSs to CBSs, as a direct result of the increase of the covered area of CBSs. Figure 8(b) illustrates the case of filtering the minimum values of $E$ obtained using the same method. Increasing the CBS cell radius will have small affect over the minimum values of energy consumption until near $R_{c} \cong 40 \mathrm{~m}$. For bigger values of $R_{c}$, it is found that minimum values of $E$ will accomplish lower values, thus, more energy conservation will be achieved due to the reduction and saving in the RF power amplifications as well as the HVAC air condition devices. Figure 8(c) illustrates the Energy Efficient Usage index $(\eta)$ versus the CBS radius. It is found that increasing $R_{c}$ will lead to an increase of the energy efficiency index, in addition, by increasing the CBS radius, there will be more energy saving (Figure 8(a)), and, gained throughput (Figure 8(b)).

\subsection{Effect of CBS Coverage Area}

Figure 9(a) shows the behavior of capacity $C$ against $R_{c}$ for different values of $d$, The results show that

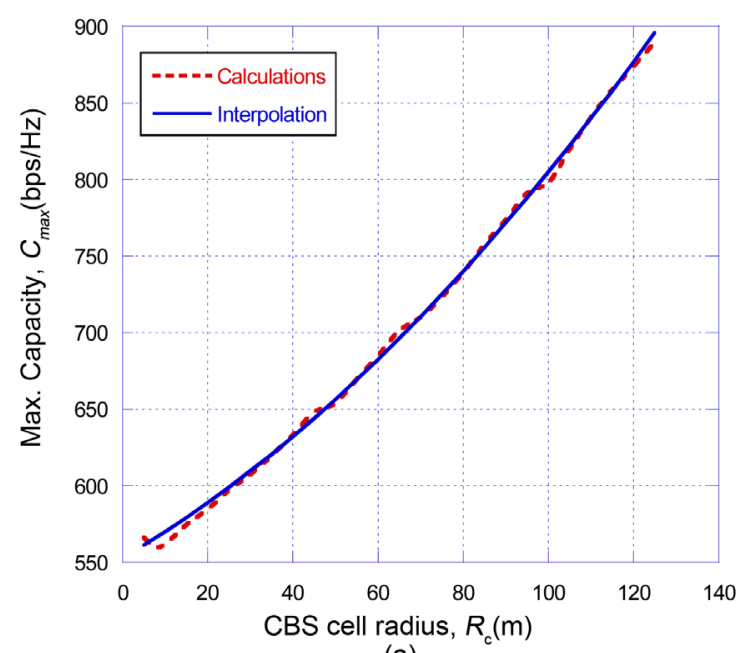

(a)

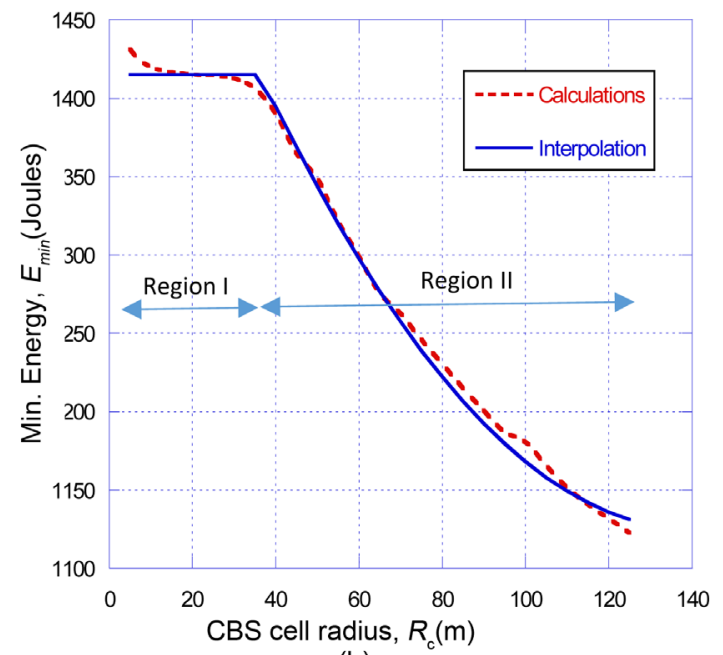

(b)

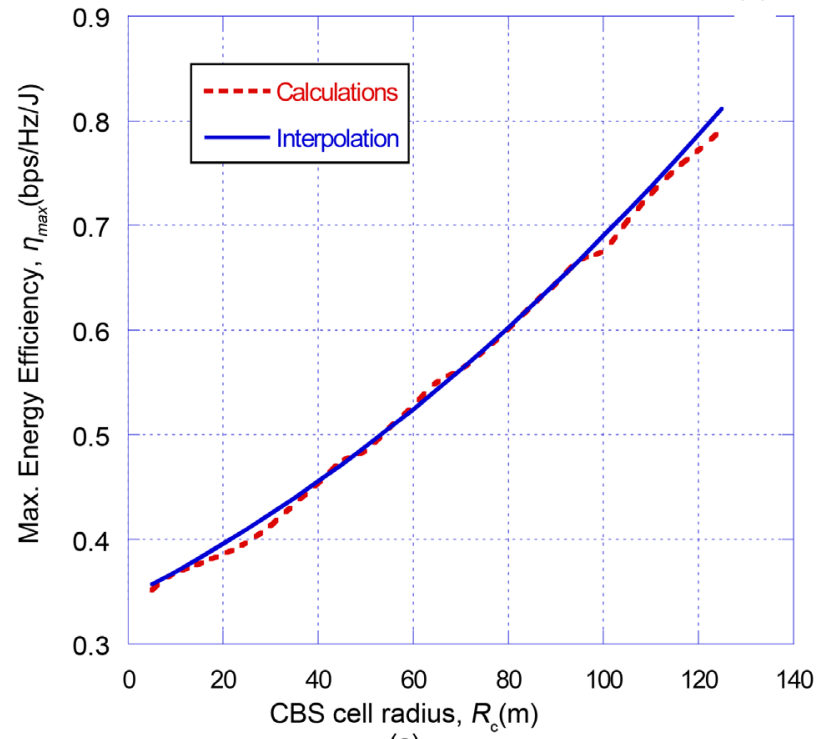

(c)

Figure 8. Shown in dotted red line, behavior ofmaxima: (a) capacity; (b) energy; (c) energy efficiency obtained for all values of inter MBS/CBSs distances versus CBS cell/coverage area radii $R_{c}$ (shown in red dotted lines), and, the reverse interpolation approximation (shown in blue lines). 


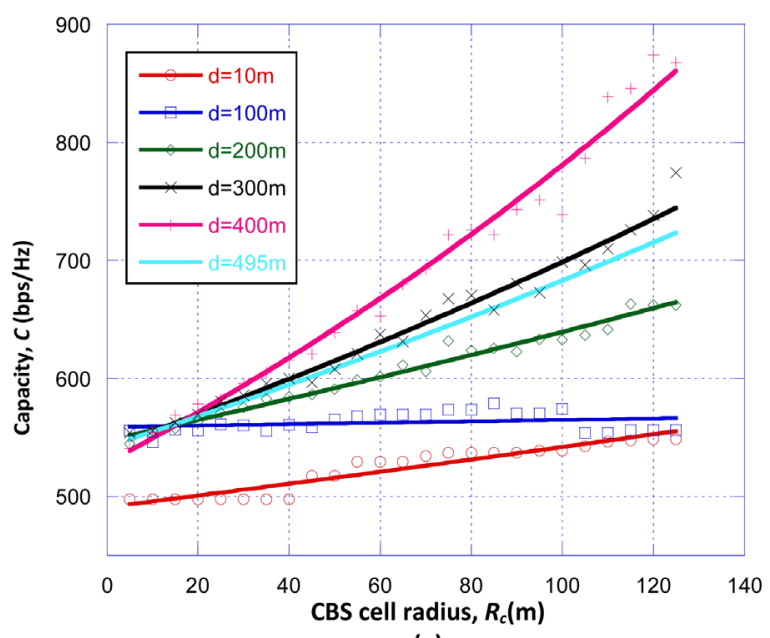

(a)

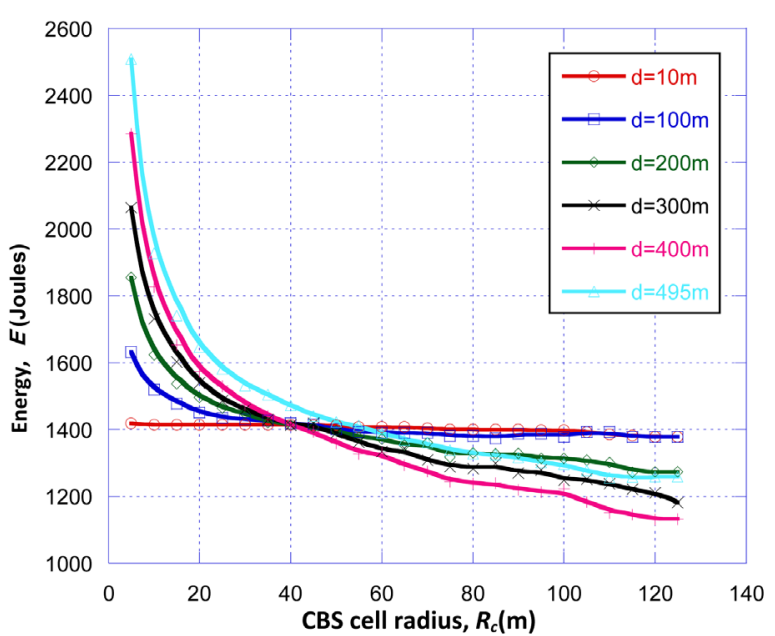

(b)

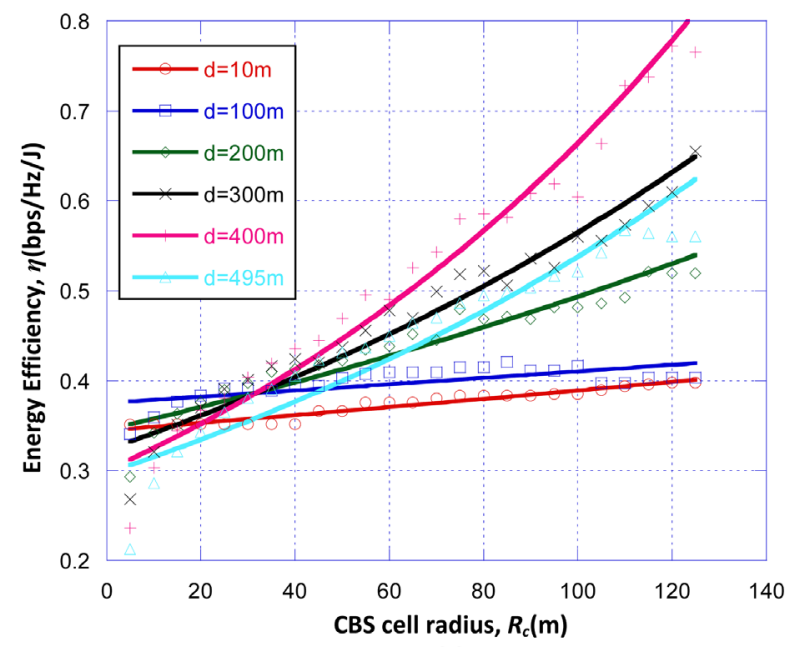

(c)

Figure 9. Behavior of: (a) capacity; (b) energy; (c) energy efficiency versus CBS cell/coverage area radii $R_{c}$ for different values of distance $d$.

$C$ increases when increasing CBS cell radius, and it is more sensitive for larger values $d$, i.e. when CBSs are far away from MBS. For very small values of $R_{c}$, and since $R_{c}$ is proportional to $d$, it is found that $C$ is in its lowest value due to the inability of the system to offer more traffic offloading or additional resources. Figure 9(b) shows the behavior of $E$ when varying $d$ for different values of $R_{c}$. It shows that for smaller values of $R_{c} \quad(<40 \mathrm{~m})$, the smaller the $d$, the larger the $E$ consumption, while, for this certain value of $R_{c} \quad(=40 \mathrm{~m})$ energy consumption is the same for all CBS located at different locations away from MBS (all $d$ ). Also, the nearer the CBS to the MBS, the less sensitivity to varying the cell radius $R_{c}$. The figure also shows that for values of $R_{c}>40 \mathrm{~m}$, the larger the $d$, the lower the Energy consumption. This means that it is preferable to use CBSs with larger values of $R_{c}$ at the far distances from MBS, while for nearer distances it is preferable to use non-overlapping with smaller coverage CBS which will allow the usage of larger number of them, which in turn, will allow more traffic offloading opportunities.

Figure 9(c) shows the behavior of $\eta$ against $R_{c}$ for different $d$ values. It shows that $\eta$ increases with the increase of $R_{c}$, and it is more sensitive to larger values of $d . \eta$ increases due to the increase of available number of CBSs deployed over the available $d$, where, as mentioned in the previous subsection: $N_{c} \cong\left\lfloor\pi d / R_{c}\right\rfloor$. The energy efficiency is found to be less sensitive at small values of $R_{c}$. To conduct further investigations over the corporate behavior of these parameters, Figure 10 shows the best obtained values w.r.t. the CBS cell radius values. These values are investigated over the range of $d$. Figure 10(a) shows the maxi- 


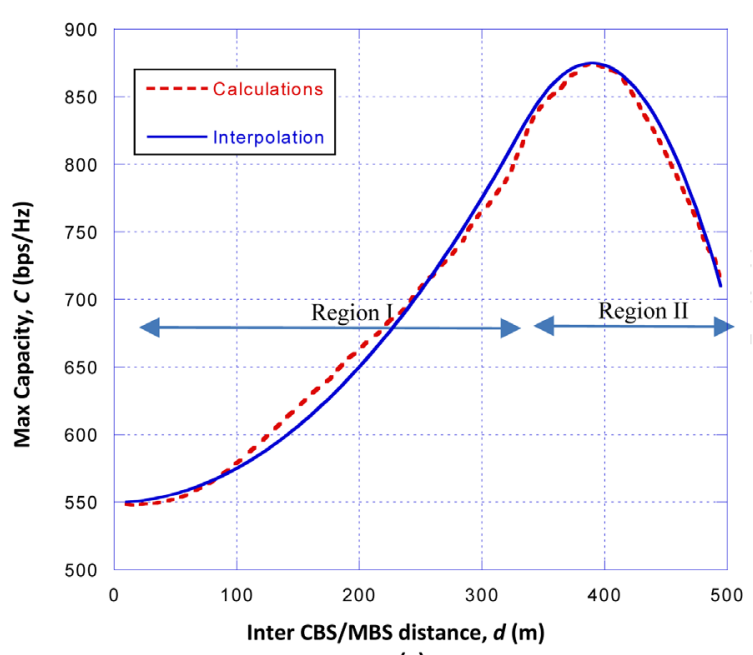

(a)

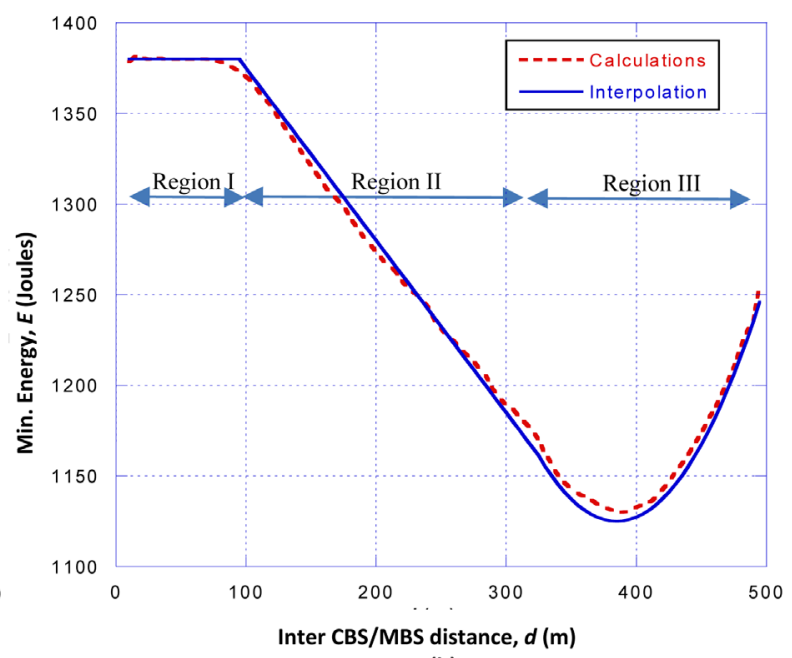

(b)

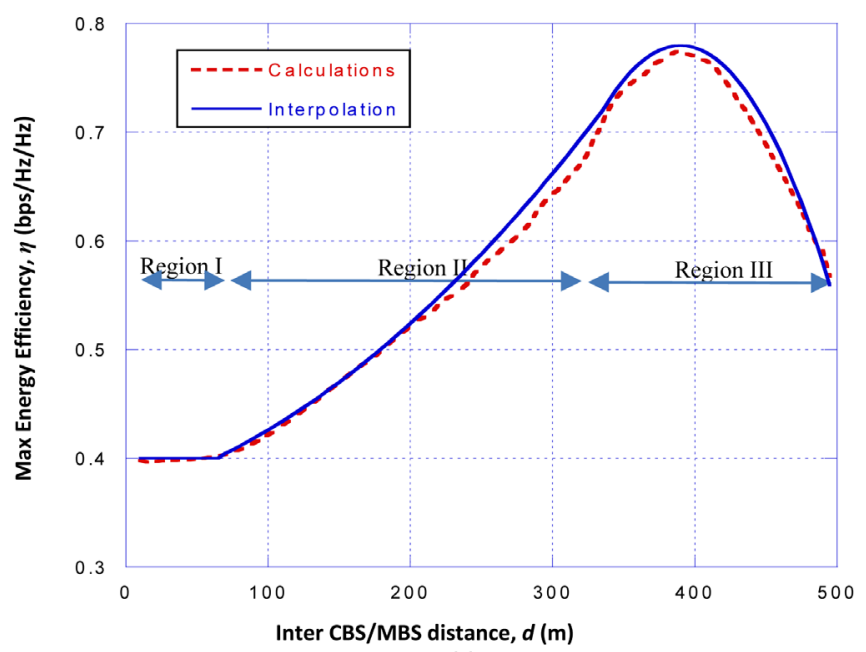

(c)

Figure 10. Shown in dotted red line, maxima behavior of: (a) capacity; (b) energy; (c) energy efficiency versus distance $d$ for different values of CBS cell/coverage area radii $R_{c}$.

mum obtained values of $C$, it is found that for nearer distances of $d$, capacity rises all the way till near the MBS cell edge, where it starts to inflict and decays again. Figure 10(b) shows the minimum consumed energy using different CBS cell radii, and for all the range of $d$. It follows an almost stable state near MBS, then it performs better energy consumption linearly all the range till near MBS cell edge (about $400 \mathrm{~m}$ ) where it inflicts and starts to lose the ability to reduce energy consumption. These behaviors corporate in the behavior of $\eta$, where it is shown in Figure 10(c) that it starts to rise till near the MBS cell edge (about 400 m) where it inflicts and starts to decay.

\subsection{Asymptotic Analysis}

To reduce the complexity of calculations to find the asymptotic behavior of the previous results, deeper analysis is conducted. A mathematical reverse interpolation of the results is obtained. A unified formula is suggested to approximate these results. This is performed in order to provide a short but effective tool to help engineers working in the field of CR field planning. The suggested formula is applied with several parameters relative to the distance used ( $d$ or $R_{c}$ ). Because of the nonlinearity behavior of these results, a suggested form of a nonlinear equation is used,

$$
y=a(x-b)^{c}+d
$$


where $a, b, c$ and $d$ are the parameters used for results reverse interpolation. Moreover, because of the nonlinearity behavior of these results, results are dealt with as multiple regions' curves, and apply different parameters to each region.

It is found that the best parameters to fit the results' interpolation are:

- maximum values of $C, E$, and $\eta$ for different values of $R_{c}$ :

$$
\begin{gathered}
C_{\max }=0.009\left(R_{c}+85\right)^{2}+480 ; \quad 5 \leq R_{c} \leq 125, \quad \text { (Figure 8(a)) } \\
E_{\max }= \begin{cases}1415, & 5 \leq R_{c} \leq 36, \quad \text { (Figure 8(b), region I) } \\
0.027\left(R_{c}-140\right)^{2}+1125 & 36 \leq R_{c} \leq 125, \quad \text { (Figure 8(b), region II) }\end{cases} \\
\eta_{\max }=1.15 \times 10^{-5}\left(R_{c}+95\right)^{2}+0.23 ; \quad 5 \leq R_{c} \leq 125, \quad \text { (Figure 8(c)) }
\end{gathered}
$$

- and, maximum values of $C, E$, and $\eta$ for different values of $d$ :

$$
\begin{gathered}
C_{\max }=\left\{\begin{array}{lll}
0.0023(d-5)^{2}+560, & 10 \leq d \leq 355, & \text { (Figure 10(a), region I) } \\
0.015(d-390)^{2}+860, & 355 \leq d \leq 500, & \text { (Figure 10(a), region II) }
\end{array}\right. \\
E_{\max }=\left\{\begin{array}{lll}
1380, & 10 \leq d \leq 95, & \text { (Figure 10(b), region I) } \\
-0.95+1470, & 95 \leq d \leq 325, & \text { (Figure 10(b), region II) } \\
0.01(d-385)^{2}+1125, & 325 \leq d \leq 500 & \text { (Figure 10(b), region III) }
\end{array}\right. \\
\eta_{\max }=\left\{\begin{array}{lll}
0.41, & 10 \leq d \leq 85, & \text { (Figure 10(c), region I) } \\
1.8 \times 10^{-6}(d+97)^{2}+0.35, & 85 \leq d \leq 320, & \text { (Figure 10(c), region II) } \\
-2 \times 10^{-5}(d-390)^{2}+0.76 & 320 \leq d \leq 500 & \text { (Figure 10(c), region III) }
\end{array}\right.
\end{gathered}
$$

\section{Conclusion}

Using a combination of an analytical model and practical simulation, several outcomes have been achieved. Using variable parameters of $d$ (average distance between CBSs and MBS) and $R_{r}$ (MBS coverage area radius), illustrated their effect on $C$ (overall system capacity), and $E$ (overall system energy consumption), and $\eta$ (Energy Efficiency). When varying $d$, the results obtained show that increasing $d$ will increase the overall system capacity $C$ till near the edge of the MBS coverage, where $C$ will start to decrease due to the decrease of the overall system SNR (due to the increase of the number of $\mathrm{CBS}^{\mathrm{s}}$ which will in turn increase the interference). Increasing $d$ and using CBSs with cell radii of less than $<40 \mathrm{~m}$ will increase the system consumption of energy, while, with cell radii $>40 \mathrm{~m}$ will be a good choice for energy consumption saving policy. The more $d$ approaches the MBS cell edge the more energy saving is obtained until an inflection point; where the system starts to reduce the energy saving. Finally the more $d$ is increased the more $\eta$ will be accomplished. Using CBSs with bigger cell radii will increase the sensitivity of $\eta$ to respond to the effect of increasing $d$. When varying $R_{r}$, the results show that the overall system capacity $C$ is more sensitive at larger $d$, while, the overall energy consumption $E$ is more sensitive and inversely proportional to $d$ at $R_{r}<40 \mathrm{~m}$ and not as sensitive and forwardly propotional to $d$ at $R_{c}>40 \mathrm{~m}$. This indicates that using smaller non-overlapping coverage area CBSs is preferable near the MBS. This work proposed several nonlinear equations with fixed coefficients to be used by field engineers to achieve the results by minimum reduced computation complexity.

\section{References}

[1] Correia, L.M., Zeller, D., Blume, O. and Ferling, Y. (2010) Challenges and Enabling Technologies for Energy Aware Mobile Radio Networks. IEEE Communications Magazine Special Issue on Green Radio, 48, 66-72. http://dx.doi.org/10.1109/MCOM.2010.5621969

[2] ITU (2014) Energy Efficiency Metrics and Measurement Methods for Telecommunication Equipment L.1310, "PrePublished Recommendations”. Telecommunication Standardization Sector of ITU.

[3] Gruber, M., Blume, O., Ferling, D., Zeller, D., Imran, M.A. and Calvanese-Strinati, E. (2009) EARTH—Energy Aware 
Radio and Network. IEEE 20th International Symposium on Personal, Indoor and Mobile Radio Communications, Tokyo, 13-16 September 2009, 1-5. http://dx.doi.org/10.1109/pimrc.2009.5449938

[4] Hossain, E., Bhargava, V.K. and Fettw, G.P. (2012) Green Radio Communication Networks. Cambridge University Press, United Kingdom. http://dx.doi.org/10.1017/CBO9781139084284

[5] EARTH (Energy Aware Radio and Network Technologies_August 2014). EARTH. https://www.ict-earth.eu/publications/deliverables/deliverables.html.

[6] Green Network Technologies INFSO-ICT-247733, Deliverable D3.2, EARTH. https://bscw.ict-earth.eu/pub/bscw.cgi/d70460/EARTH_WP3_D3.2.pdf.

[7] FP7-ICT-2009-4-247733-EARTH Book (2012) EU Funded Research Project—January 2010 to June 2012, EARTH (Energy Aware Radio and Network Technologies). https://www.ict-earth.eu.

[8] FP7 in Brief (2014) Office for Official Publications of the European Communities, Luxembourg. http://ec.europa.eu/research/fp7/pdf/fp7-inbrief_en.pdf.

[9] 7th Framework Programme for Research and Technological Development (2014) European Commission, Research \& Innovation. http://ec.europa.eu/research/fp7/understanding/fp7inbrief/funding-schemes_en.html

[10] Magnus Olsson, E.A. (2012) A Methodology to Evaluate Radio Network Energy Efficiency at System Level. 1st ETSI TC EE Workshop, Genoa, Italy.

[11] TR 36.814, Further Advancements for E-UTRA Physical Layer Aspects RAN1 (2010) 3GPP in 2 EPS. http://www.in2eps.com/3g36/tk-3gpp-36-814.html.

[12] Auer, G., Giannini, V., Godor, I., Skillermark, P., Olsson, M., Imran, M.A., et al. (2011) Cellular Energy Efficiency Evaluation Framework. Proceedings of the IEEE 73rd Vehicular Technology Conference, Yokohama, 15-18 May 2011, 1-6. http://dx.doi.org/10.1109/VETECS.2011.5956750

[13] Gür, G., Bayhan, S. and Alagöz, F. (2013) Energy Efficiency Impact of Cognitive Femtocells in Heterogeneous Wireless Networks. Proceedings of the 1st ACM Workshop on Cognitive Radio Architectures for Broadband-CRAB'13, Miami, 4 October 2013, 53-60. http://dx.doi.org/10.1145/2508478.2508480

[14] Bayhan, S. and Alagoz, F. (2012) Scheduling in Centralized Cognitive Radio Networks for Energy Efficiency. IEEE Transactions on Vehicular Technology, 62, 582-595. http://dx.doi.org/10.1109/TVT.2012.2225650

[15] Goldsmith, A. (2005) Wireless Communication. Cambridge University Press, Cambridge. http://dx.doi.org/10.1017/CBO9780511841224

[16] Nandy, S.C. Voronoi Diagrams. Advanced Computing and Microelectronics Unit, Indian Statistical Institute, Kolkata 700108. http://www.tcs.tifr.res.in/ ghosh/subhas-lecture.pdf

[17] Miu, A. (2001) Lecture 7: Voronoi Diagrams. 6.838 Computational Geometry. http://nms.lcs.mit.edu/ aklmiu/6.838/L7.pdf

[18] FP7 Project Management (2014) Eurescom Archive Website, July 2014. http://archive.eurescom.eu/services/project management/default.asp

[19] Abate, Z. (2009) WiMax RF Systems Engineering. Artech House, Boston and London.

[20] Gallager, R.G. (2001) Claude E. Shannon: A Retrospective on His Life, Work, and Impact. IEEE Transactions on Information Theory, 47, 2681-2695. http://dx.doi.org/10.1109/18.959253

[21] ETSI TR 136913 V9.0.0, LTE; (2010-2012) Requirements for Further Advancements for Evolved Universal Terrestrial Radio Access (E-UTRA) (LTE-Advanced). Centre, 3GPP Mobile Competence.

[22] D6.13.7: Test Scenarios and Calibration Cases Issues 2. (2006) World Initiative New Radio (WINNER II), Deliverable, IST-4-027756.

[23] Requirements Related to Technical Performance for IMT-Advanced Radio Interface(s), M.2134, Report ITU-R. (2008). https://www.itu.int/dms_pub/itu-r/opb/rep/R-REP-M.2134-2008-PDF-E.pdf 
Scientific Research Publishing (SCIRP) is one of the largest Open Access journal publishers. It is currently publishing more than 200 open access, online, peer-reviewed journals covering a wide range of academic disciplines. SCIRP serves the worldwide academic communities and contributes to the progress and application of science with its publication.

Other selected journals from SCIRP are listed as below. Submit your manuscript to us via either submit@scirp.org or Online Submission Portal.
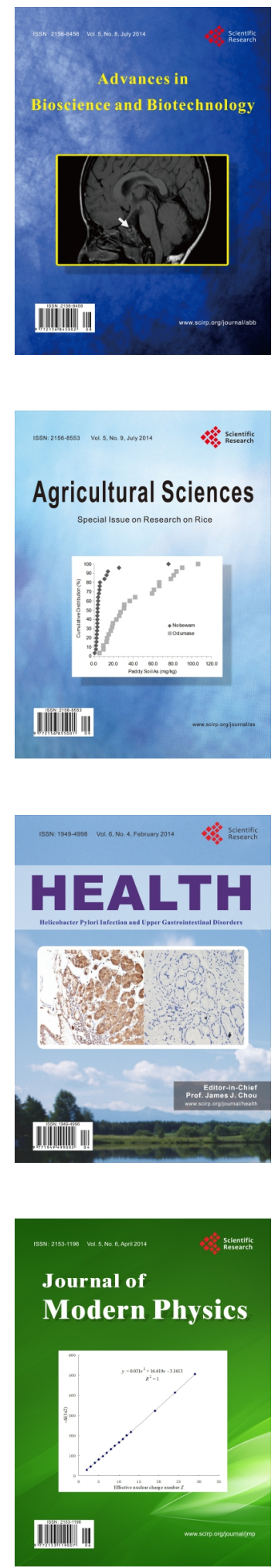
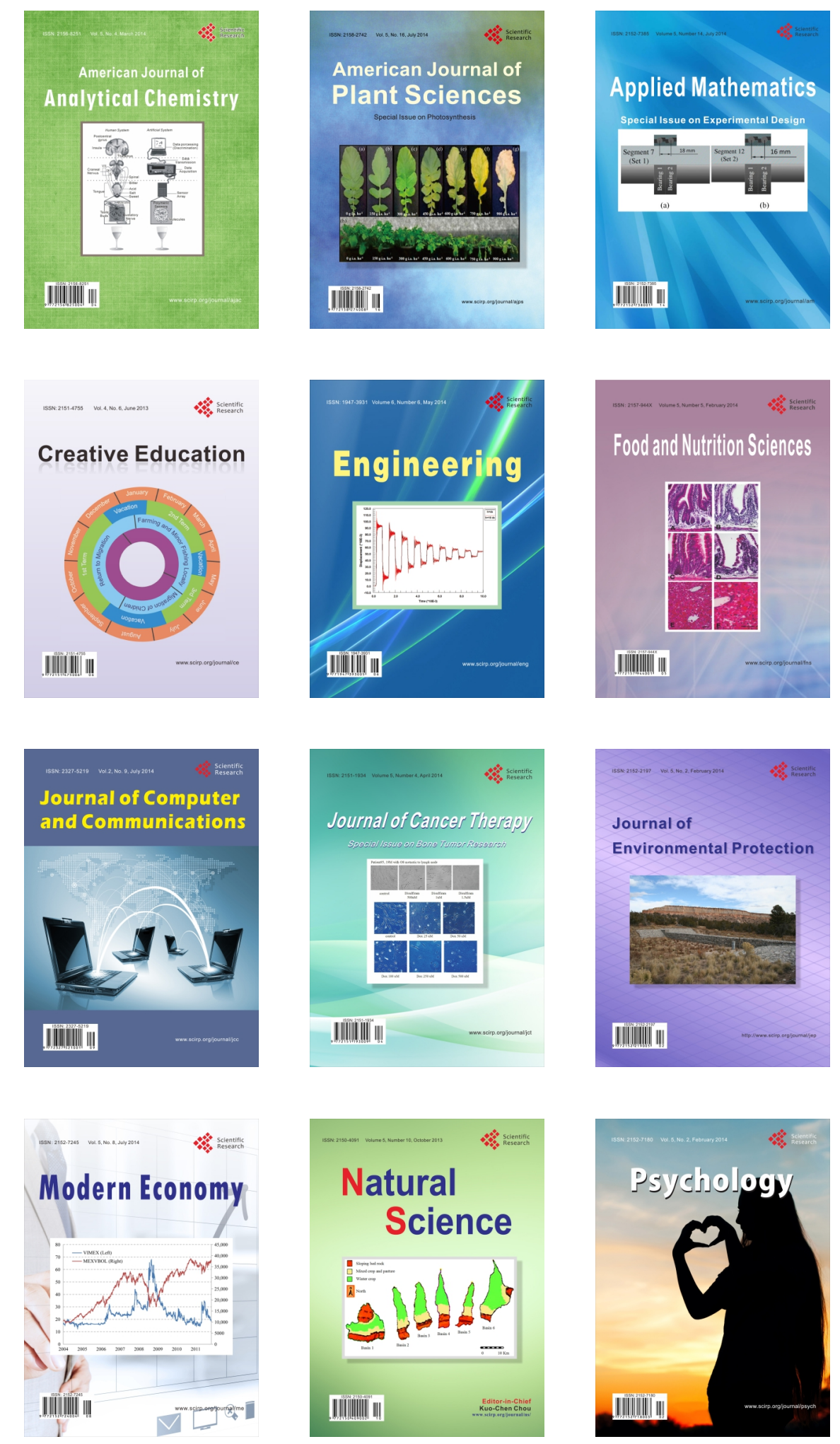